\title{
Natural Therapy Advisory System based on Fusion of Image Processing and Text Processing
}

\author{
A. M. Ali \\ Dept. of Orthopedics \\ Faculty of Medicine \\ Mansoura, Egypt
}

\author{
A. E. Amin \\ Dept. of Computer Science \\ Faculty of Specific Education \\ Mansoura, Egypt
}

\author{
A. F. Abdo \\ Dept. of Computer Science \\ Faculty of Specific Education \\ Mansoura, Egypt
}

\begin{abstract}
This paper presents an advisory system that helps those in charge of cases of physical therapy in order to a diagnosis of patients and providing appropriate treatment steps for each case. The proposed system consists of three main stages namely image processing, text processing and advisory system. Each of these stages is contributed as the following:
\end{abstract}

Image processing stage is used two algorithms to achieved its task, the first is Gray-Level Co-Occurrence Matrix (GLCM) algorithm to extract the features from $\mathrm{x}$-ray images and the second is KNN algorithm to classified the images features, then stored in a database.

Text processing stage is used to convert the text report corresponding to an image to automated reports. This occurs through several steps like preprocessing, tokenization, stop responding word filtering, and count frequency.

Lastly, the advisory system is consists of two parts, a knowledge base is built by expert man that helps to extract the rules from databases of image and text. The second part is inference engine that executes the matching between query rule and knowledge base.

The results of this paper are so convincing, where the proposed system is applied on several patients and the extracted of cure reports are satisfied to experts in the field.

\section{Keywords}

Image processing, Text processing, Advisory system

\section{INTRODUCTION}

\subsection{Image processing}

An image is an artifact that depicts visual perception, for example a two-dimensional picture that has a similar appearance to some subject - usually a physical object or a person, thus providing a depiction of it [1].

Images may be two-dimensional, such as a photograph, screen display, and as well as a three-dimensional, such as a statue or hologram [1]. An image is an array, or a matrix, of square pixels (picture elements) arranged in columns and rows [2]. There are different types of images depends on the degree of gray and these types X-ray images under this type [3].

$\mathrm{X}$-rays image- describes radiation which is part of the spectrum which includes visible light, gamma rays and cosmic radiation [3].

There are areas uses images to it in a way where he began science image processing.

Image Processing, with the development of the internet, and the availability of image capturing devices such as digital cameras, image scanners, the size of digital image collection is increasing rapidly. A large collection of images is referred to as image database. An image database is a system where image features are integrated and stored [2].As a subcategory or field of digital signal processing; digital image processing has many advantages over analog image processing [3].

Image processing is using mathematical operations by using any form of signal processing such as region based techniques and boundary based techniques [4]. A region based technique uses whole shape region but a boundary based technique only uses boundary points of shapes in feature vector extraction. Some commonly used Fourier based signatures are complex coordinate (CC), polar coordinate (PC), angular function $(\mathrm{AF})$, triangular area representation (TAR), chord length distance (CLD) and angular radial coordinate (ARC) [4].

Multi-user technique helped in image processing in the image retrieval process after the store as an example of a large, one of the most important methods of image retrieval.

Content Based Image Retrieval (CBIR) is the task of retrieve the images from the large collection of database on the basis of their own visual content as shown as figure 1. CBIR is used for automatic indexing and retrieval of images depending upon contents of images known as features [5].

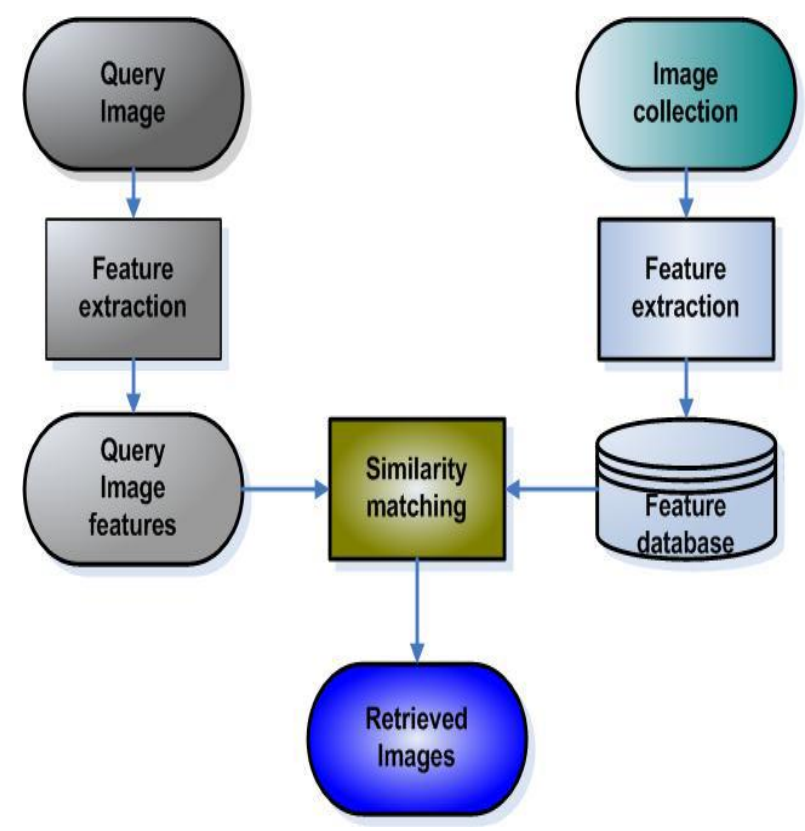

Figure 1 Block diagram of Content Based Image Retrieval [5]

Feature extraction is a start point in CBIR where is an initial set of measured data and builds derived values (features) intended to be informative and non-redundant, facilitating the subsequent learning and generalization steps, and in some 
cases leading to better human interpretations. Feature extraction is related to dimensionality reduction.

Feature extraction is a general term for methods of constructing combinations of the variables to get around these problems while still describing the data with sufficient accuracy.

Though texture plays a significant role in image analysis and pattern recognition, only a few architectures implement onboard textural feature extraction [3].

Content-based retrieval methods can be classified into classes depending on the features they use such as: color, texture, and shape [3].

Each features class is further divided into subclasses by the type of the algorithm used for the constructing the feature vector [2]. Many methods for feature extractions are used such as:

- Color Features [2]

- Texture Features [2]

- $\quad$ Shape Features

In statistical texture analysis, texture features are computed from the statistical distribution of observed combinations of intensities at specified positions relative to each other in the image. According to the number of intensity points (pixels) in each combination, statistics are classified into first-order, second-order and higher-order statistics. The Gray Level Cooccurrence Matrix (GLCM) method is a way of extracting second order statistical texture features [3].

The GLCM features are: the energy, contrast, correlation and the homogeneity. The first GLCM texture feature is calculated by using the following formula [4]:

\subsubsection{The energy formula}

$$
\text { Energy }=\sum_{i, j} p(i, j)^{2}
$$

Where:

$\mathrm{i}, \mathrm{j}$ are a single pixel.

$\mathrm{p}(\mathrm{i}, \mathrm{j})$ is the probability that two pixels with a specified separation have grey levels $i$ and $j$.

\subsubsection{The contrast formula

$$
\text { Contrast }=\sum_{i, j}|i-j|^{2} p(i, j)
$$

\subsubsection{The correlation formula}

$$
\text { Correlation }=\sum_{i, j} \frac{\left(i-\mu_{i}\right)\left(j-\mu_{j}\right) p(i, j)}{\sigma_{i} \sigma_{j}}
$$

\section{Where}

$\mu \mathrm{i}, \mu \mathrm{j}$ represents the horizontal and the vertical means in the matrix.

$\mu \mathrm{i}$ and $\mu \mathrm{j}$ represent standard deviation.

$$
\begin{aligned}
& \text { 1.1.4 The homogeneity formula } \\
& \text { Homogeneity }=\sum_{i, j} \frac{p(i, j)}{1+|i-j|}
\end{aligned}
$$

After extracting features are stored in the database and are classified features of the images as a result of the large size of the data stored in the database.

\subsection{Image Classifications}

Classification includes a broad range of decision-theoretic approaches to the identification of images. All classification algorithms are based on the assumption that the image in question depicts one or more features and that each of these features belongs to one of several distinct and exclusive classes. The classes may be specified a priori by an analyst or automatically clustered into sets of prototype classes, where the analyst merely specifies the number of desired categories [5].

Classification have closely related objectives, as the former is another form of component labeling that can result in segmentation of various features in a scene.

Image classification analyzes the numerical properties of various image features and organizes data into categories. Classification algorithms typically employ two phases of processing: training and testing. In the initial training phase, characteristic properties of typical image features are isolated and, based on these, a unique description of each classification category, training class, is created. In the subsequent testing phase, these feature-space partitions are used to classify image features [6].

The description of training classes is an extremely important component of the classification process. In supervised classification, statistical processes or distribution-free processes can be used to extract class descriptors [5]. Unsupervised classification relies on clustering algorithms to automatically segment the training data into prototype classes. In either case, the motivating criteria for constructing training classes are that they are [6]:

- Independent, a change in the description of one training class should not change the value of another,

- Discriminatory, different image features should have significantly different descriptions, and

- Reliable, all image features within a training group should share the common definitive descriptions of that group.

There are various methods for image classification; some of these methods are explained in the following section [7]:

- $\quad$ K-Nearest-Neighbor

- Decision Tree

- Naïve Bayesian

- Artificial Neural Network

- Support Vector Machine

- K-Means

$\mathrm{K}$ - Means is one of the simplest unsupervised learning algorithms that solve the well-known clustering problem. The procedure follows a simple and easy way to classify a given data set through a certain number of clusters (assume $\mathrm{k}$ clusters) fixed a priori. Flow chart of K-Means is show in Figure 2 


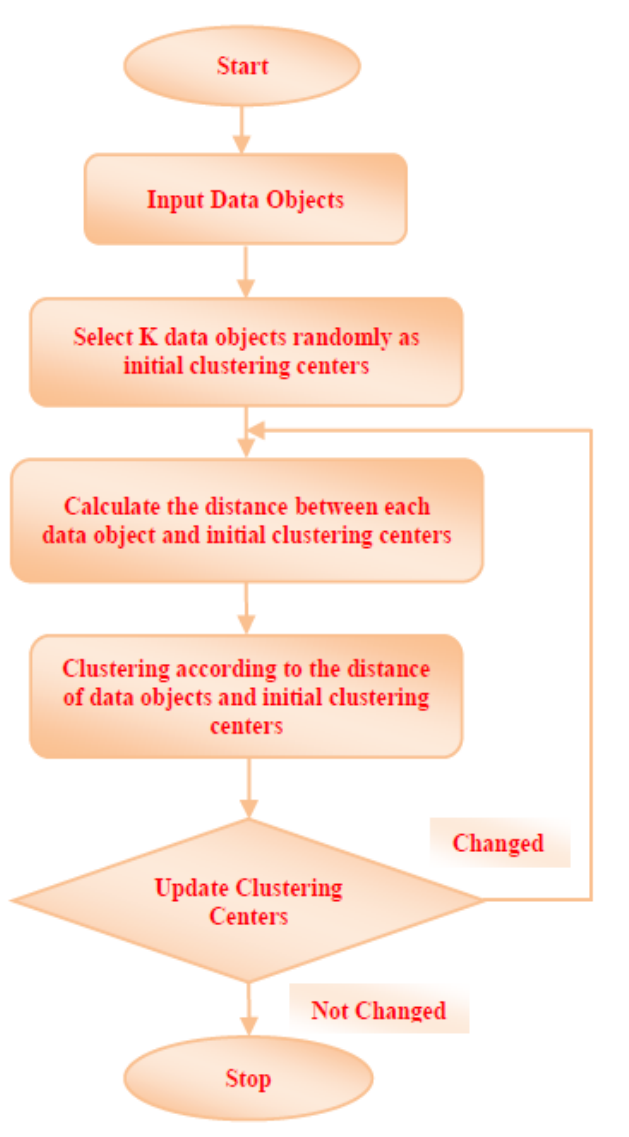

Figure 2 K-Means Flow Chart [10]

\subsection{Text Processing}

Text data mining is useful for research into any reports because it gives researchers the ability to automatically detect event in any discipline. We use the text data to detect event in this step and our method is depicted in figure 2 [9].

Input Text, convert the string to a list of tokens based on whitespace. This process also removes punctuation marks from the text.

Stop word filtering, eliminate the words which are common and their presence does not tell us anything about the dataset, such as: the, and, for, etc.

Stemming filtering, reduce each word to its stem, removing any prefixes or suffixes

Indexing, can be index the data after filtering and stemming by using TF-IDF which is a weighting scheme that weighs features in tweets based on how often the word occurs in an individual tweet compared with how often it occurs in other tweets. Then by measuring the weight for each keyword, we can decide the related event [9].

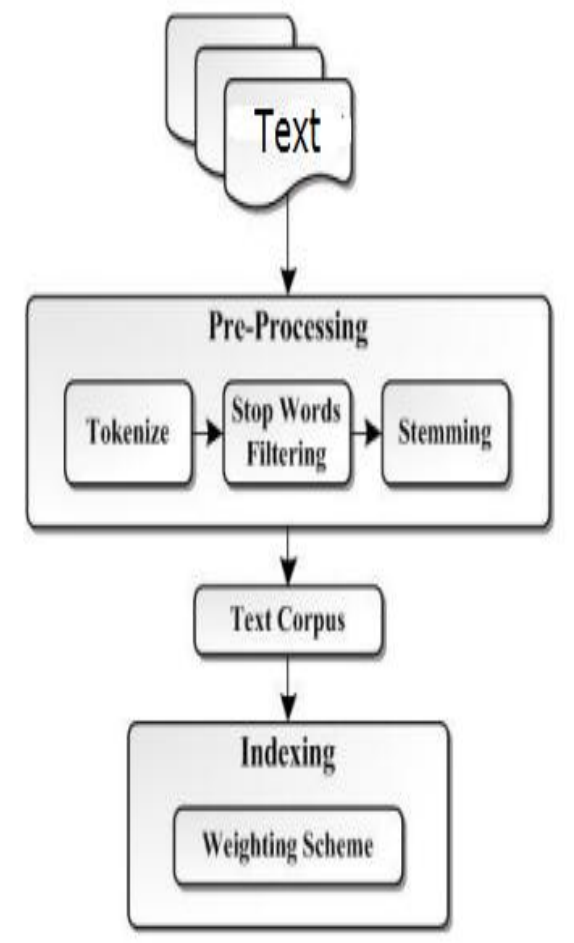

Figure 3 Block diagram of text data [9]

In case of classification, where there are two possible classes \{positive, negative\}, a classification algorithm creates a classifier on the basis of a training set. In the bagging method, it creates a series of classifiers. These classifiers are combined into a "compound classifier". The final prediction of the "compound classifier" is gained from weighted combination of individual classifier predictions. The meaning of this theory can be described as a "voting procedure" where the objective is to find the classifier which is having stronger influence on the final prediction than other classifiers [8].

The aim of this paper is to develop advanced algorithms for text detection, especially for text and image in Specialist Report data. The algorithm will be used in advance to fuse different features that can be extracted from x-ray data.

\subsection{Fusion Image and Text Data}

For multimedia data, we apply fusion for text and image by combining text and image features. It uses our database that contains both text and photos to the proposed event mining system which consists of event keyword detection, event photo classification photo selection. The features we extract in this step are the combination of features in section text, and section image. In our fusion method, if the report text mining score is less than a threshold, this means that the text mining is not reliable so the report is classified using the image only; otherwise, the report is classified using the text or image [9].

\section{PROPOSED SYSTEM}

The proposed system consists of three main units namely: image processing unit, text processing unit, and advisory system unit as shown in figure 4 . 


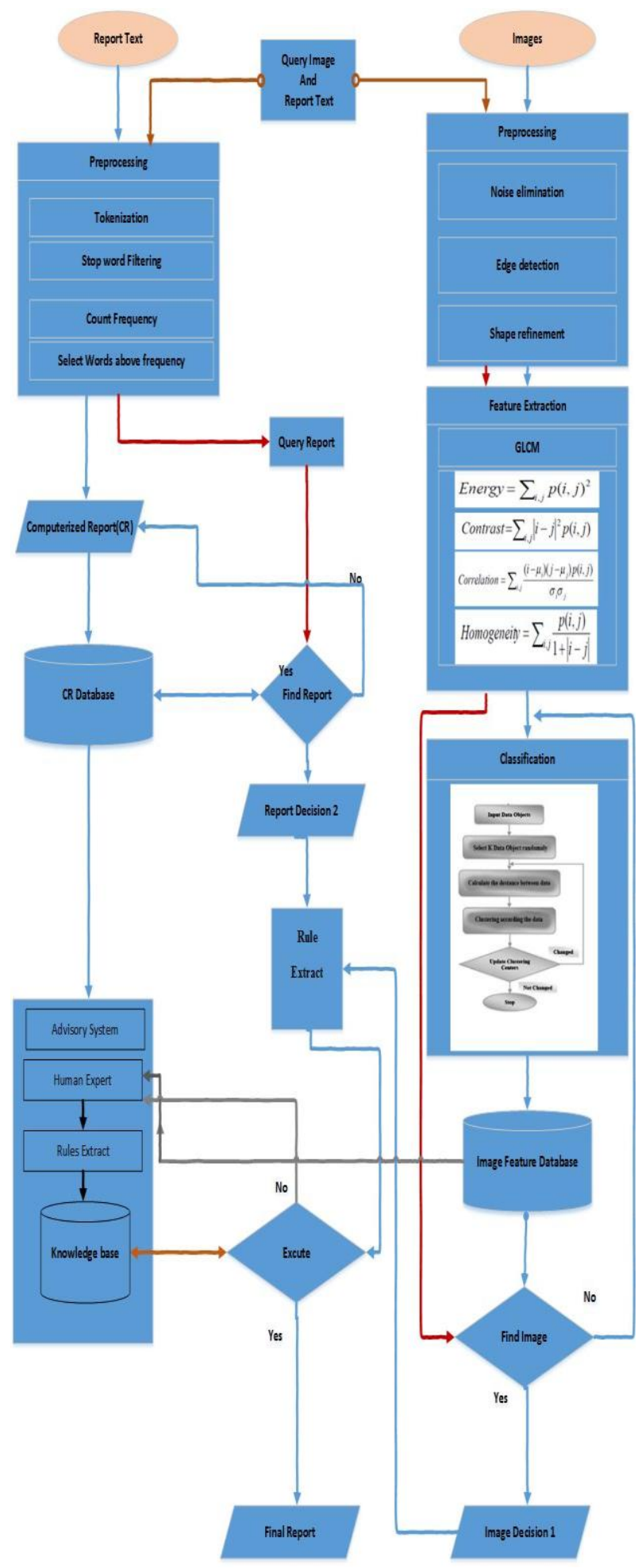

Fig. 4 Flow Chart of Advisory System

Both of image and text processing units are used to extract the main features for image and text. Image processing is used to produce a classified features database. Whereas text processing is used to produce a computerized report, stored in corresponding database (CR database).

The advisory system is consists of three stage, firstly human expert that help to building rules from image decision and text report decision. Secondly rules are extracted from two decisions as shown in Table1.
Table 1: Extract and Execute Rules Example

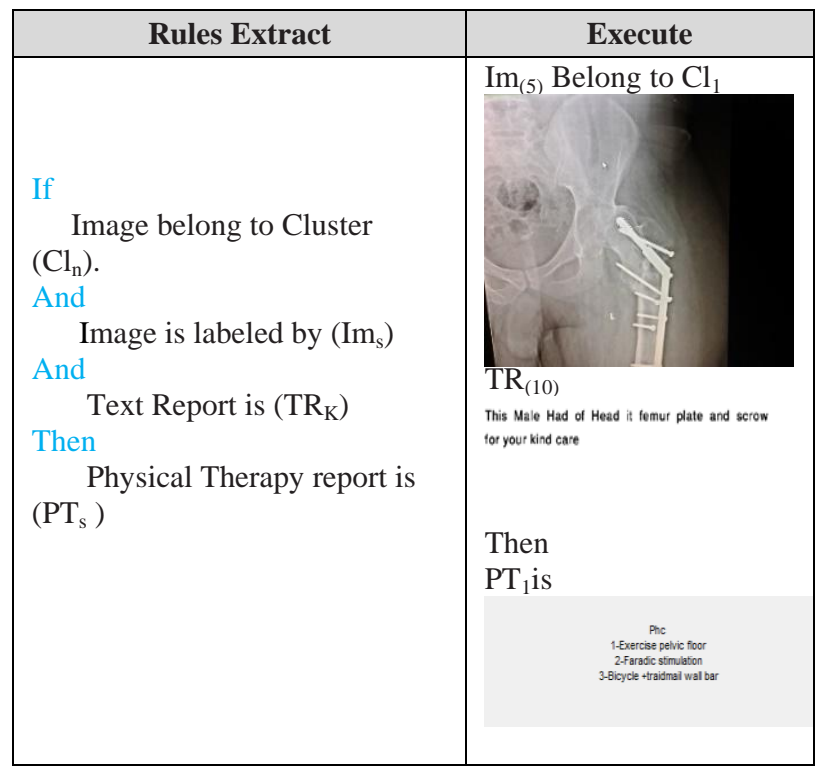

Lastly, extracted rules are stored in a knowledge base.

\section{EXPERIMENTAL AND RESULTS}

To evaluate the proposed system, samples from X-Rays image with corresponding text reports had been collected as shown in table 2 .

Table 2: Some Patient's Image and Report

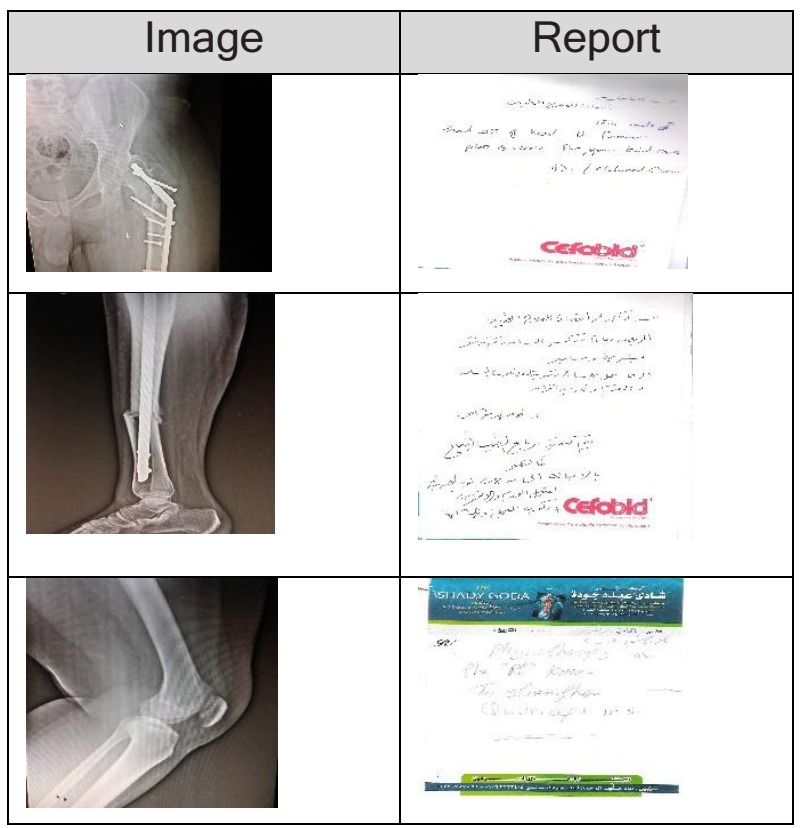

The registration in the proposed system is achieved by inputting personal data of patient's as shown in figure 5 . 


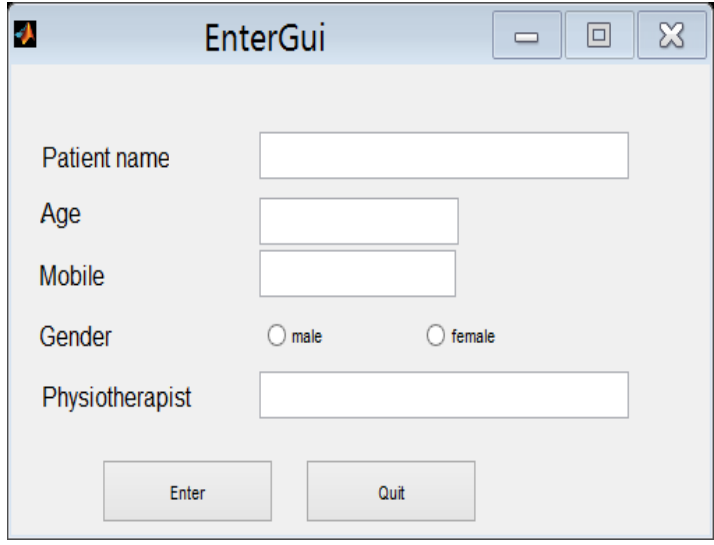

Fig. 5 Login to Proposed System

Images and Corresponding Text Reports are fed to the proposed system, then both of them take its paths consecutively to extract its features. Images features are extracted from image processing stage as shown in figure 6 . The image's features are stored in images database. Figure 7 illustrate assertion message for storing of image features successfully.

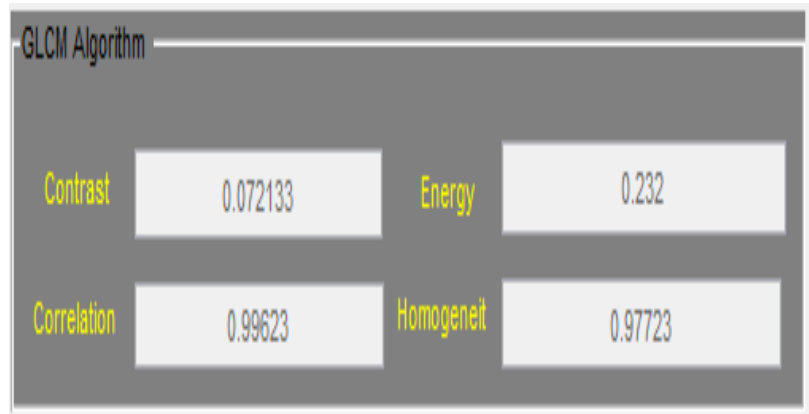

Fig.6 Image features extraction example

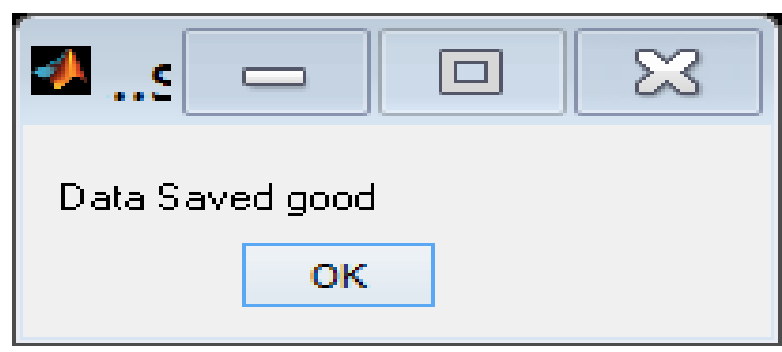

Fig. 7: Assertion message for image's features

Image's features are classified into categories according to their distinctive features as shown in Figure 8. So; it's easier to retrieve the image during the query. The proposed system confirms the completion of the classification process, by showing a message to the user as shown in Figure 9.

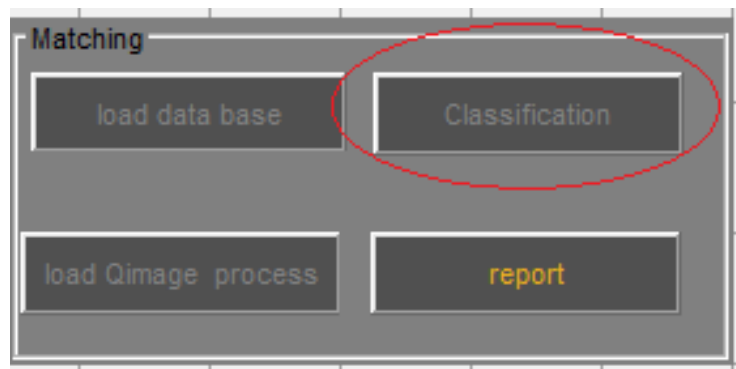

Fig. 8 Classification Button

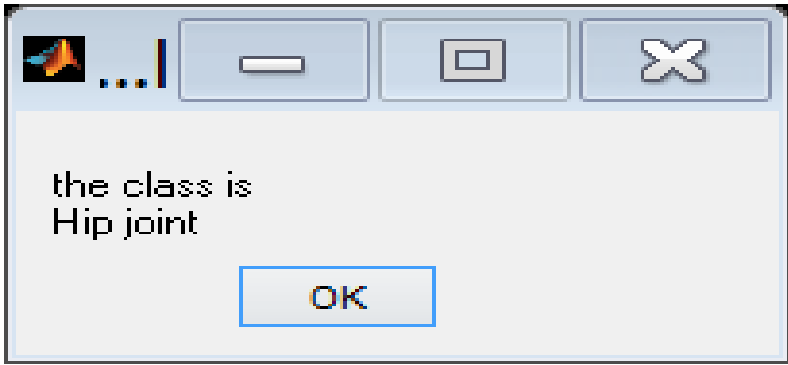

Fig. 9 Assertion Classification message

The corresponding report for each image is addressed during the second stage namely text processing. In this stage, the text report is converted to automated report by passing through several steps, in turn, are: preprocessing, tokenization, stop responding word filtering, count frequency. So is getting saved a computerized report as shown in Figure 10.

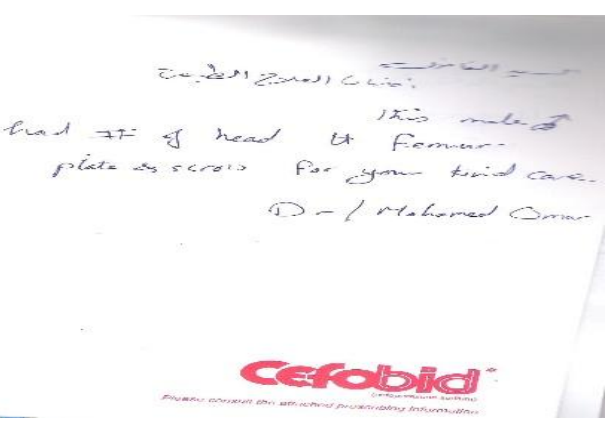

This Male Had of Head it femur plate and scrow for your kind care

Fig. 10: convert text Report to computerized report.

In the third phase, the advisory part is achieved by placing the knowledge base rules. These rules are used both of expert men and the databases of image's features and automated text reports.

When the query about treatment steps for Patients case based on x-ray images and the attached report, is fed to the proposed system and extract features of the image and convert the report to the computerized report, as previously explained.

The rule is derived from both of the image and the report decisions. The matching between the query rule and the knowledge base of the proposed advisory system determines the method of treatment as shown in Figure 11. 


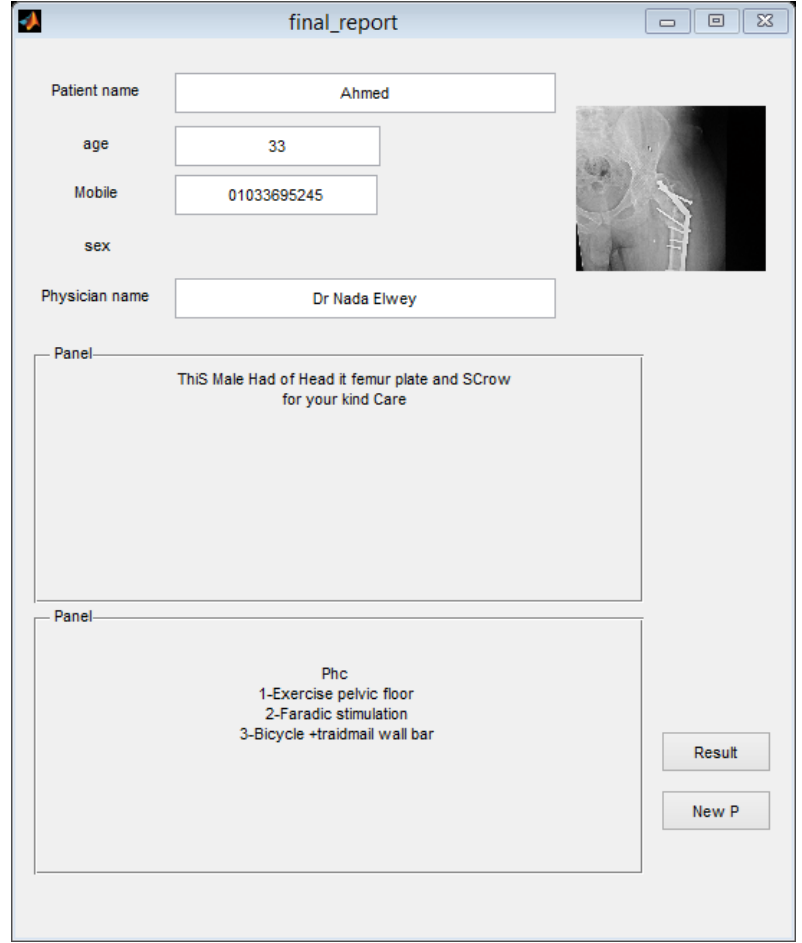

Fig. 11: Final Treatment Steps Report

\section{CONCLUSIONS}

This paper presents the submission of a proposal to diagnose types of bone fractures and the development of a method of treatment of an advisory system that based on image processing and text processing. In the image processing stage, the proposed system is fed by images (x-ray), which used for extraction of their own features that are classified according to their distinctive and stored in a database. In the second stage, text processing will be fed by the corresponding text report and used to convert it to automated report. Then the results which stored in databases will be displayed on the expert that helped in the formation of the knowledge base.

The results which were extracted from the proposed system are showed that it's convincing where, many of the patient's cases are applied and extract reports of treatment steps, which matched with the experts in the field.

\section{REFERENCES}

[1] Sonya Eini and Abdolah Chalechale,"Shape Retrieval Using Smallest Rectangle Centroid Distance", International Journal of Signal Processing, Image Processing and Pattern Recognition, 2013, PP. 61-68.

[2] André Aichert, Feature extraction techniques, CAMP MEDICAL SEMINAR WS0708,2008,PP. 1-8.

[3] P. Mohanaiah, P. Sathyanarayana, L. GuruKumar, Image Texture Feature Extraction Using GLCMApproach, International Journal of Scientific and Research Publications, Volume 3, Issue 5, May 2013,PP. $1-5$.

[4] ChitwanBhalla Surbhi Gupta, A Review on Splicing Image Forgery Detection Techniques, IRACST International Journal of Computer Science and Information Technology \& Security (IJCSITS), Vol.6, No.2, Mar-April 2016, ISSN: 2249-9555.

[5] A.Ramesh Kumar, D.Saravanan, Content Based Image Retrieval Using ColorHistogram, A.Ramesh Kumar et al, (IJCSIT) International Journal of Computer Science and Information Technologies, Vol. 4 (2) , 2013, 242 - 245.

[6] S. Yan, X. Xu, D. Xu, S. Lin, and X. Li. Beyond spatial pyramids: A new feature extraction framework with dense spatial sampling for image classification. In Proc. ECCV, 2012, pages 473-487.

[7] Md. Khalid Imam Rahmani1 and Naina Pal, Kamiya Arora, Clustering of Image Data Using K-Means and Fuzzy K-Means, (IJACSA) International Journal of Advanced Computer Science and Applications, Vol. 5, No. 7, 2014, PP.160-163.

[8] Feilong Cao, BoLiu and Dong Sun Park., " Image classification based on effective extreme learning machine", Neurocomputing, Vol.102, 2013, PP. 90-97.

[9] Samar M. Alqhtani, Suhuai Luo and Brian Regan, FUSING TEXT AND IMAGE FOR EVENTDETECTION IN TWITTER, The International Journal of Multimedia \& Its Applications (IJMA) Vol.7, No.1, February 2015,PP. 27-35. 